\title{
The Recent Evolution of Early-Type Galaxies as Seen in their Cold Gas
}

\author{
Lisa M. Young \\ Physics Department, New Mexico Tech, 801 Leroy Place, Socorro NM, 87801, USA \\ email: lyoung@physics.nmt.edu
}

\begin{abstract}
I present an overview of new observations of atomic and molecular gas in early-type galaxies, focusing on the ATLAS ${ }^{3 \mathrm{D}}$ project. Our data on stellar kinematics, age and metallicity, and ionized gas kinematics allow us to place the cold gas into the broader context of early-type galaxy assembly and star formation history. The cold gas data also provide valuable constraints for numerical simulations of early-type galaxies.
\end{abstract}

Keywords. galaxies: elliptical and lenticular, cD - galaxies: evolution - galaxies: formation

\section{Introduction}

Two of the major interrelated challenges of galaxy formation are understanding the basic origin of the Hubble sequence (spiral vs. elliptical and lenticular galaxies) and the present-day dichotomy in galaxy colors (the red sequence vs. the blue cloud or starforming sequence). Most early-type (elliptical and lenticular) galaxies are poor in cold gas; yet their stars did form, albeit a long time ago, in a precursor that was probably gas-rich and blue. In this context, then, the story of what happened to the cold gas in those precursors is a crucial part of the evolution of early-type galaxies. The current gas contents of early-type galaxies can offer valuable quantitative benchmarks for numerical simulations of galaxy evolution.

Early-type galaxies probably moved onto the red sequence as they lost their cold gas to consumption (star formation), AGN and star formation feedback, and environmental processing in groups and clusters. On the other hand, they could also have acquired cold gas through cooling out of a hot medium and through mergers. Their cold gas supports some current star formation activity, and the rates and efficiencies of that process (in comparison to the behaviors of spirals) can offer some useful constraints on star formation in general. These are some of the issues addressed through observations of nearby earlytype galaxies in the ATLAS ${ }^{3 \mathrm{D}}$ project.

\section{Sample and Observations}

The ATLAS ${ }^{3 \mathrm{D}}$ sample is a complete volume-limited sample of early-type galaxies nearer than $42 \mathrm{Mpc}$ and brighter than $M_{K}=-21.5$, i.e. having stellar masses greater than $M_{\star}=10^{9.9} \mathrm{M}_{\odot}$ (Cappellari et al. 2011a). The early-type galaxy sample is actually drawn from a parent sample which has no color or morphological selection, and the 260 galaxies lacking spiral structure form the basis of the project. The core of the observations consists of integral-field optical spectroscopy over a field of at least $33^{\prime \prime} \times 41^{\prime \prime}$. For these nearby galaxies, $80 \%$ of the sample has spectroscopic coverage out to $0.8 R_{e}$ or farther (Emsellem et al. 2011). While the wavelength coverage of the spectra is not large by current standards, the data do offer stellar kinematics, [O III], $\mathrm{H} \beta$ and occasionally other emission lines, and line indices sensitive to age, metallicity, and $\alpha$ enhancement. 
This is all useful for inferring both the star formation and the assembly histories of the galaxies, in addition to the ionized gas excitation and kinematics.

ATLAS $^{3 \mathrm{D}}$ galaxies primarily inhabit the red sequence, as expected, though a small percentage are blue, especially at masses $M_{\star}<10^{10.5} \mathrm{M}_{\odot}$ (Cappellari et al. 2011a; Young et al. 2014). A discussion of their morphological characteristics is found in Cappellari et al. (2011b). Basically, the majority of early-type galaxies are disky, fast rotating, oblate galaxies, and relatively few, primarily at high masses, are spherical or triaxial and slowly rotating (Krajnović et al. 2011; Emsellem et al. 2011; Weijmans et al. 2014). The twodimensional kinematic data highlight the incidence of multiple kinematic subcomponents, decoupled or even counterrotating cores, and kinematic twists within the galaxies (see especially Krajnović et al. 2011). Ongoing work also involves deep optical imaging (Duc et al. 2011) to give better limits on recent interactions.

An initial CO survey with IRAM 30m telescope probed the molecular content in the central $20^{\prime \prime}$ of the ATLAS ${ }^{3 \mathrm{D}}$ galaxies, giving detection limits in the range $10^{7}$ to $10^{8}$ $\mathrm{M}_{\odot}$ (Young et al. 2011). Some more exotic high density molecular tracers have also been observed in a few cases (Bayet et al. 2012; Crocker et al. 2012; Bayet et al. 2013; Davis et al. 2013). Alatalo et al. (2013a) present interferometric CO observations of most of the $\mathrm{CO}$ detections, at a typical spatial resolution of $5^{\prime \prime}(300 \mathrm{pc}$ to $1 \mathrm{kpc})$ over a $1^{\prime}(5-11$ kpc) field of view. Serra et al. (2012) also present interferometric H I observations of 170 of the ATLAS $^{3 \mathrm{D}}$ galaxies from the Westerbork array; those data cover a $30^{\prime}$ field and a velocity range of $4000 \mathrm{~km} \mathrm{~s}^{-1}$ at typical resolutions of $35^{\prime \prime}$, and they have the sensitivity to detect $\mathrm{H}$ I column densities of a few $10^{19} \mathrm{~cm}^{-2}$. For a modest-sized cloud of gas this corresponds to mass limits of $10^{6}$ to $10^{8} \mathrm{M}_{\odot}$.

\section{Abundant Cold Gas in Early-Type Galaxies}

From the observations noted above, the detection rate of molecular gas in massive early-type galaxies is $22 \% \pm 3 \%$ (Young et al. 2011). To place this value in context it is important to remember that the sample selection is not biased by far-IR detections or even by selection in the $B$ band, as were many previous surveys in early-type galaxies. The $\mathrm{H}_{2}$ masses fall in the range $10^{7.1}$ to $10^{9.3} \mathrm{M}_{\odot}$, or $\log \mathrm{M}\left(\mathrm{H}_{2}\right) / \mathrm{M}_{\star}$ in the range -3.5 to -1.1 . For the galaxies with CO maps, the molecular gas is usually concentrated in the central kpc or so (Davis et al. 2013) in disks, rings, bars, and irregular configurations (Alatalo et al. 2013a). The detection rate of $\mathrm{H}$ I is $40 \%$ outside of the Virgo Cluster; H I masses range from $10^{6.3} \mathrm{M}_{\odot}$ to $10^{10} \mathrm{M}_{\odot}$, and the atomic gas is found in isolated clouds, regular disks (sometimes many tens of $\mathrm{kpc}$ in diameter) and disturbed configurations (Serra et al. 2012).

The ATLAS ${ }^{3 \mathrm{D}}$ project thus has the largest set of cold gas maps ever assembled for earlytype galaxies. Atomic and/or molecular gas in quantities of $10^{7}$ to $10^{9} \mathrm{M}_{\odot}$ is surprisingly common in these galaxies, being found in about $50 \%$ of them. Often the gas-rich galaxies are detected in both phases, but not always; and the galaxies have values of $\mathrm{M}\left(\mathrm{H}_{2}\right) / \mathrm{M}(\mathrm{H}$ I) that range from $10^{-2}$ to $10^{2}$, even considering just the ones detected in both phases.

\section{Which kinds of ETGs have cold gas?}

Figure 1 shows a color-magnitude diagram of the ATLAs ${ }^{3 \mathrm{D}}$ galaxies, and it demonstrates that molecular gas detection rates are still strong among red sequence galaxies, particularly at high stellar masses where the star formation rates are not large enough to move the galaxies off the red sequence in integrated colors. Young et al. (2014) have also discussed the reddening effects of dust, which are modest. Careful statistical analysis 


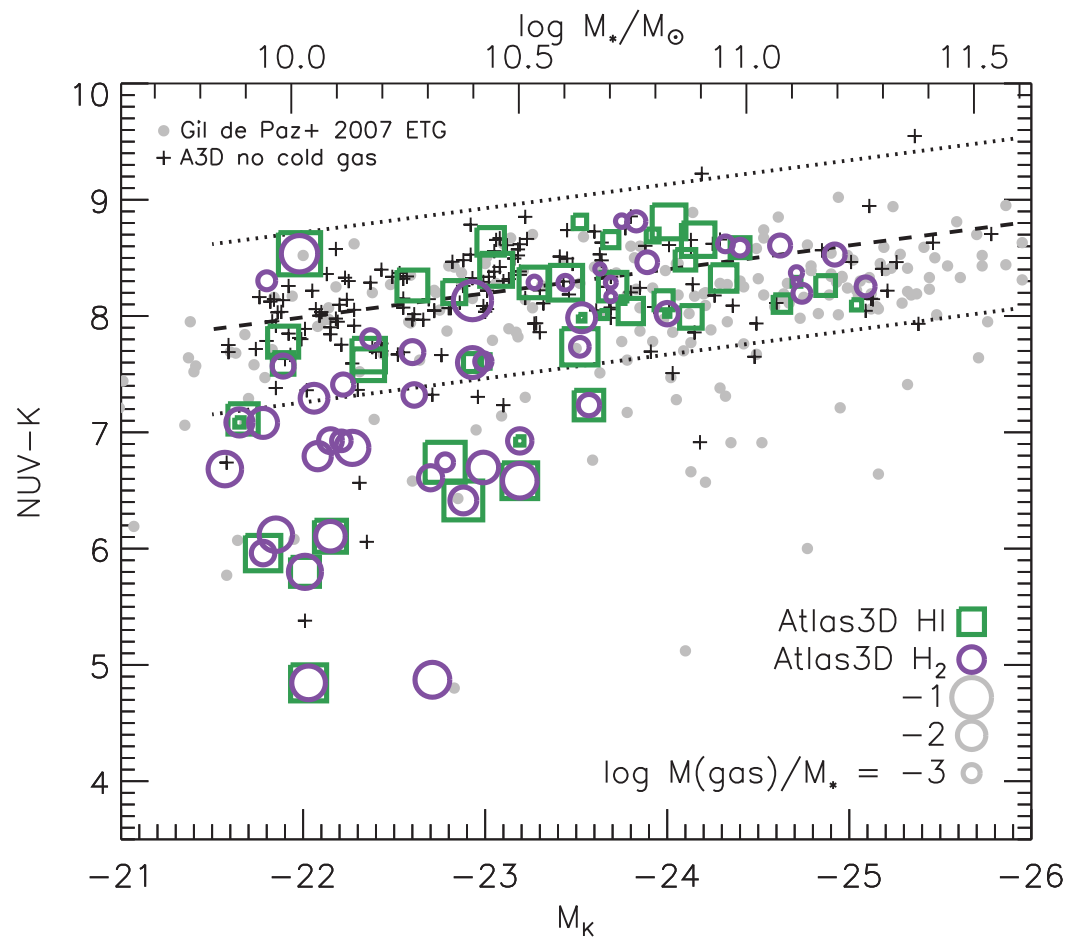

Figure 1. NUV-K color-magnitude diagram for the ATLAS ${ }^{3 \mathrm{D}}$ sample. The dashed line is the red sequence ridge line and the dotted lines are $2 \sigma$ redder and bluer, as described in Young et al. (2014). $\mathrm{H}$ I and $\mathrm{H}_{2}$ masses are indicated by the symbol sizes.

shows that the molecular mass detection rate is not a function of stellar mass over the observed ranges, nor is the $\mathrm{H}_{2}$ mass distribution function (Young et al. 2011).

While the molecular masses of early-type galaxies do not correlate with stellar mass, they do correlate with structural parameters. Cappellari et al. (2013b) show that, at a fixed stellar mass, the early-type galaxies with low stellar velocity dispersion and large effective radius (i.e. with more dominant disky stellar components rather than spheroids) have larger molecular masses. It is tempting to assume that this might be because the molecular gas disk gives birth to a young, disky stellar component. And while that is partly true, the currently observed molecular masses are usually only $1 \%$ of the stellar masses, or less, so the young stellar disks are probably too small to drive the wholegalaxy bulge/disk ratios. They must be driven by older stellar disks. In addition, as we will discuss below, much of the molecular gas is kinematically misaligned with respect to the stars.

There are some interesting differences in the atomic and molecular contents of earlytype galaxies. Krajnović et al. (2011) show that about $86 \%$ of early-type galaxies are the oblate fast rotators. These galaxies can grow through gas-rich minor mergers and associated in situ star formation, or through recent major mergers with the appropriate geometry to preserve a large net angular momentum (Naab et al. 2013). They are detected at about the same rate in both CO and H I (Serra et al. 2012). More rare are the slow rotators, which can be formed from recent mergers with special geometries ensuring near-cancellation of the net angular momentum, and the spherical non-rotators, which are proposed to grow through a large number of isotropically oriented minor mergers (Naab et al. 2013). Slow- and non-rotators are rarely detected in CO but are detected in 
$\mathrm{H}$ I at about the same rate as the fast rotators are detected in H I (Serra et al. 2012). This information suggests that at least in some cases the origin of the atomic gas is different from that of the molecular gas, or perhaps the galaxy structure affects the formation or longevity of the molecular phase. Sarzi et al. (2013) also discusses some effects of the galaxy structure on the hot gas content of the ATLAS ${ }^{3 \mathrm{D}}$ galaxies.

We conclude that the quenching of a galaxy and the approach to the red sequence do not always involve the loss of all, or even most, of the cold gas from an early-type galaxy. Alternatively, perhaps some cold gas is reacquired during or after the approach to the red sequence. The presence of molecular gas in an early-type galaxy is strongly connected to the presence of a dynamically cold, disky stellar component, and the connection may be partly (but not entirely) due to the formation of a young stellar disk out of said molecular gas. Contrary to this, atomic gas is more indiscriminate, being found in approximately equal quantities in both the disky fast rotators and the bulge-dominated slow rotators, and often not associated with any notable star formation.

\section{Angular Momenta as clues to the history of the gas}

Early-type galaxies have much more interesting and diverse stellar kinematics in their inner regions than spirals do - as is abundantly clear from the examples shown in Krajnović et al. (2011). The comparisons between gas and stellar kinematics are also important clues to the evolution of these galaxies. For example, Davis et al. (2011) plot the misalignment angle between stellar and ionized or molecular gas kinematics; the distribution peaks at zero but has a long flat tail all the way to $180^{\circ}$ (retrograde gas). Stellar-gas kinematic misalignments are common in molecular and ionized gas, occurring in at least $35 \%$ of early-type fast rotators (see also Sarzi et al. 2010). Similarly, half of all $\mathrm{H}$ I-detected fast rotators have that $\mathrm{H}$ I kinematically misaligned with respect to the stars (Serra et al. 2014). These papers argue that gas at misalignments $>30^{\circ}$ cannot be simply recycled material from internal stellar mass loss, because it has the wrong kinematics. Perhaps it could be brought in to the galaxy in an accreted satellite, or perhaps (as discussed below) it cooled out of hot gas at large radii in the galaxy halo, where the hot gas is vulnerable to many external torques. If these arguments apply to all of the misaligned gas plus some portion of the aligned gas as well, they apply to something like $\gtrsim 50 \%$ of the cold gas in nearby early-type galaxies.

There is also some evidence for external gas accretion from the metallicity measurements. The ATLAS ${ }^{3 \mathrm{D}}$ sample galaxies follow a well-established stellar mass-metallicity relation, with a few notable outliers at markedly low metallicity (but enhanced $\alpha$-element abundances) for their mass. These also tend to be blue and rich in molecular gas. McDermid et al. (2014) postulate that these are cases in which low metallicity gas was accreted, so the population of younger stars with lower metallicity is biasing the "average" metallicity estimate.

\section{Comparison to simulations}

Past work on simulating the cold gas content of galaxies has mainly focused on the gas-rich spiral galaxies (e.g. Lagos et al. 2011). The simulations of early-type galaxies are more challenging if for no other reason than the quantities of cold gas are generally smaller, requiring higher fidelity. Serra et al. (2014) made high resolution re-simulations of dark matter halos extracted from a larger $\lambda$ CDM simulation, implementing gas cooling, star formation and supernova feedback. Cooling is not carried out to molecular gas

temperatures, but $\mathrm{H}$ I is converted to $\mathrm{H}_{2}$ by fiat at surface densities of $10 \mathrm{M}_{\odot} \mathrm{pc}^{-2}$. Not 
surprisingly, the details of the feedback prescription have strong influence on the final gas distribution and kinematics, and even in some cases on the stellar kinematics. There is significant tension between these simulations and the observations in the sense that the simulations produce too much prograde cold gas and not enough misaligned cold gas.

Lagos et al. (2014) describe a semi-analytical model which follows the quantities of cold gas that cooled out of the hot halo, were brought in by mergers, returned to the ISM from internal stellar mass loss, and consumed by star formation. They stress the co-evolution of the hot and cold gas, emphasizing that an improved treatment of gentle ram pressure stripping of the hot halo is necessary to reproduce the observed cold gas contents of early-type galaxies. A work in preparation also addresses the kinematic misalignments of the cold gas in early-type galaxies; there are not enough minor mergers to explain all of the misaligned gas, but cooling out of a hot halo might contribute misaligned cold gas if the hot halo has been torqued so that it is also misaligned with the stellar body of the galaxy (Lagos 2014, private communication).

\section{Star formation and galaxy dynamics}

For some years it has been suspected that the empirical star formation "laws" (predicting the star formation rate produced by a given quantity of cold gas) might be somewhat different in early-type galaxies than in spirals, due to the systematically different gravitational potentials (e.g. Kawata et al. 2007; Martig et al. 2009). Indeed, hydrodynamical simulations of Martig et al. (2013) found that the molecular gas in spherical galaxies should not reach the same high local densities as the molecular gas in spirals, and they predicted something like factors of 2 lower star formation efficiencies in early-type galaxies. These kinds of factors of two could prove to be important as simulations of galaxy evolution move into precision mode.

Interestingly, Davis et al. (2014) observe that the star formation efficiency of early-type galaxies correlates with a measurement of whether most of the gas is in the rising or the flat part of the rotation curve. Specifically, where most of the molecular gas is in the rising part, the efficiency is lower by factors of a few. These types of observations offer the opportunity to make empirical tests of the star formation "laws" beyond the spiral galaxies where they were developed, so they should help refine the prescriptions used in simulations.

\section{Summary}

There is more cold gas in early-type galaxies than most of us expected. Approximately $50 \%$ of massive early-type galaxies $\left(\mathrm{M}_{\star} \gtrsim 10^{10} \mathrm{M}_{\odot}\right)$ contain $10^{7}$ to $10^{9} \mathrm{M}_{\odot}$ of $\mathrm{H} \mathrm{I}$ and/or $\mathrm{H}_{2}$. The atomic and molecular phases are not always found together; for example, Virgo Cluster members are more likely to be detected in $\mathrm{H}_{2}$ but not in $\mathrm{H}$ I.

Because many of these cold-gas-rich early-type galaxies are found on the red sequence, and the red colors are not solely the effect of dust associated with the cold gas, we infer that 'red sequence' and 'cold gas' are not mutually exclusive properties; the approach to the red sequence does not always involve the loss of all cold gas. Alternatively, some red sequence galaxies may reacquire gas after going onto the red sequence.

Much of the cold gas in early-type galaxies is kinematically misaligned with respect to the stars. Because of the misalignment it is apparently not a simple recycling of gas from stellar mass loss directly back into a cold gas phase. It remains to be seen - and would be fascinating to know - how the cold gas is aligned (or not) with respect to the hot gas, as it becomes increasingly obvious that hot and cold phases probably do 
interact with each other. We have posited that a significant portion of the cold gas in early-type galaxies is accreted either with incoming satellite galaxies in minor mergers, or has cooled from the intergalactic medium or a hot halo.

The cold gas contents and kinematics of early-type galaxies are thus shown to be sensitive probes of their evolution. The diversity in the stellar kinematics, gas contents, and gas kinematics of early-type galaxies emphasizes the diversity in their evolutionary paths. Even the diversity in the star formation properties of early-type galaxies can help improve our understanding of the star formation process. These features are now beginning to give useful constraints on numerical simulations.

Additional work on the hot gas in early-type galaxies is needed, especially regarding the thermal and dynamical interactions between hot and cold gas. More insights to the evolution of early-type galaxies should also come from the metallicities of all the gas phases.

\section{Acknowledgements}

I acknowledge support from NSF AST 1109803 and travel support from the IAU; I also thank Dr Paul T. P. Ho for the invitation to spend a sabbatical year at ASIAA.

\section{References}

Alatalo, K., Davis, T. A., Bureau, M., et al. 2013, MNRAS, 432, 1796

Bayet, E., Davis, T. A., Bell, T. A., Viti, S., 2012, MNRAS, 424, 2646

Bayet, E., et al. 2013, MNRAS, 432, 1742

Cappellari, M., et al. 2011, MNRAS, 413, 813 (Paper I)

Cappellari, M., et al. 2011, MNRAS, 416, 1680 (Paper VII)

Cappellari, M., McDermid, R. M., et al. 2013, MNRAS, 432, 1862

Crocker, A. F., Krips, M., Bureau, M., et al. 2012, MNRAS, 421, 1298

Davis, T. A., Alatalo, K., Sarzi, M., et al. 2011, MNRAS, 417, 882

Davis, T. A., Bureau, M., et al. 2013, MNRAS, 429, 534

Davis, T. A., Bayet, E., Crocker, A., Topal, S., Bureau, M., 2013, MNRAS, 433, 1659

Davis, T. A., Young, L. M., et al. 2014, MNRAS, 444, 3427

Duc, P.-A., Cuillandre, J.-C., Serra, P., et al. 2011, MNRAS, 417, 863

Emsellem, E., et al. 2011, MNRAS, 414, 888

Kawata, D., Cen, R., Ho, L. C. 2007, ApJ, 669, 232

Krajnović, D., et al. 2011, MNRAS, 414, 2923

Lagos, C. d. P., Baugh, C. M., Lacey, C. G., et al. 2011, MNRAS, 418, 1649

Lagos, C. d. P., Davis, T. A., Lacey, C. G., et al. 2014, MNRAS, 443, 1002

Martig, M., Bournaud, F., Teyssier, R., \& Dekel, A., 2009, ApJ, 707, 250

Martig, M., Crocker, A. F., Bournaud, F., et al. 2013, MNRAS, 432, 1914

McDermid, R. M., et al. 2014, MNRAS, submitted

Naab, T., Oser, L., Emsellem, E., et al. 2013, MNRAS, 444, 3357

Sarzi, M., et al. 2010, MNRAS, 402, 2187

Sarzi, M., et al. 2013, MNRAS, 432, 1845

Serra, P., et al. 2012, MNRAS, 422, 1835

Serra, P., Oser, L., Krajnović, D., et al. 2014, MNRAS, 444, 3388

Weijmans, A.-M., et al. 2014, MNRAS, 444, 3340

Young, L. M., et al. 2011, MNRAS, 414, 940

Young, L. M., et al. 2014, MNRAS, 444, 3408 